\title{
LE SMI entre ses difficultés et les pistes de réforme
}

\author{
Zineb OTMANI ${ }^{1}$, Radouane RAOUF ${ }^{2}$
}

${ }^{1}$ Laboratory of Economic Analysis and Modelling (LEAM) FSJES Souissi, UM5S, Rabat, Morocco
${ }^{2}$ Laboratory of Economic Analysis and Modelling (LEAM) FSJES Souissi, UM5S, Rabat, Morocco

\begin{abstract}
Résumé : Dans la perspective de créer et d'augmenter les richesses des nations, l'économie mondiale a connu un essor considérable grâce à la perpétuelle évolution du cadre des relations économiques et monétaires. Cependant, le financement de toutes les activités économiques, dépendantes principalement de la stabilité du système monétaire mondial, s'avère être compliquée. Depuis plusieurs décennies, un nombre important de travaux et d'approches s'intéressant au SMI cherchent à identifier les raisons des nombreuses crises financières qui ont ébranlé les économies du monde en pointant du doigt les défaillances du système monétaire et en relançant à chaque fois le débat sur sa réforme. Certains travaux évoquent l'existence des Global Imbalances comme une explication possible de la formation d'instabilités, en faisant référence aux niveaux record des déséquilibres en compte courant sur le plan international qui ont cours depuis les années quatre-vingt-dix et jusqu'à la crise contemporaine. En effet, la crise de 2008 a eu un impact direct sur le SMI et a mis en lumière un certain nombre de vulnérabilités passées jusque-là inaperçues. Néanmoins, il n'est pas question d'expliquer l'impact des crises sur le SMI mais à montrer, plutôt, que l'architecture du SMI est peutêtre un facteur provocateur des crises.
\end{abstract}

Mots-clés : Système monétaire international, réforme, régime de change, économie mondiale.

\section{Introduction}

$\mathrm{Au}$ sortir de la deuxième guerre mondiale l'économie mondiale a connu un essor considérable grâce au développement des relations économiques internationales, c'est-à-dire les mouvements massifs des flux de biens et de services, de main-d'œuvre et de capitaux et d'idées. Cependant il faut bien financer toutes ces activités économiques dépendantes principalement de la stabilité du système monétaire mondial, chose qui s'avère être compliquée par le fait que la plupart des pays ont leur propre monnaie et que les règles régissant les transactions financières varient beaucoup d'un pays à l'autre. Le système monétaire international (SMI) est généralement défini comme un ensemble de pratiques, de règles et 
d'institutions visant à organiser et surveiller les échanges monétaires et les flux financiers entre les pays. Les relations monétaires internationales ont connu une alternance de périodes où ont prévalu, tour à tour, une sorte d'organisation hiérarchisée des devises constitutives d'un $\mathrm{SMI}^{1}$ et les antagonismes nationaux liés à des relations économiques instables. Cette alternance a résulté de la difficulté à concilier la convertibilité à taux fixe, la mobilité des capitaux vecteurs d'intégration économique et financière, et l'indispensable priorité aux choix nationaux pour la définition des politiques monétaires. Sans omettre que les crises économiques en cascade qui ont ébranlé les économies du monde, tel que la crise mexicaine de 1994, la crise asiatique de 1997, la crise bancaire au Brésil en 1997-1998 et la crise financière turque en 2000, ont pointé du doigt les défaillances du système monétaire et relançaient à chaque fois le débat sur sa réforme. En effet, la crise qui a submergé l'économie mondiale en 2008 a pris par surprise la plupart des experts et des responsables politiques, elle a eu un impact direct sur le SMI en mettant en avant ses faiblesses de fonctionnement et un certain nombre de vulnérabilités passées jusque-là inaperçues, en affectant gravement les conditions de liquidité, les régimes de taux de change mis en place, la position relative des pays par rapport aux autres, etc. Cette crise a davantage mis en évidence les liens étroits entre les économies et les marchés financiers du monde, un choc subi par un grand pays se propage rapidement à l'ensemble du système. La coordination mondiale des politiques de relance budgétaire et monétaire et l'injection massive de liquidités ont évité un effondrement encore plus spectaculaire de l'activité économique dans le monde. Dès lors l'économie mondiale a été soumise à d'importants bouleversements structurels, dans la sphère financière, une série de réformes ont été adoptées et sont mises en œuvre, d'autres sont en cours d'examen, notamment la réforme du système monétaire international, surtout avec l'accentuation des pressions en vue d'une réforme du FMI et des autres institutions internationales en liaison avec les inquiétudes relatives aux déséquilibres mondiaux.

L'objectif du papier est de décortiquer les anomalies du SMI actuel dit hybride pour explorer et examiner quelques pistes de réformes envisageables, c'est dans ce sens que nous ouvrons notre discussion par un retour sur l'évolution historique du Système monétaire international (section 2). Nous verrons, ensuite, le portrait du système actuel (section 3). Pour procéder à une analyse de ses forces et ses faiblesses (section 4) afin d'étudier les différentes pistes et perspectives de réforme pour un système monétaire international qui réponds aux exigences du contexte économique actuel (section 5) avant de conclure (section 6).

\section{Retour sur l'évolution du SMI}

Le cadre des relations monétaires, comme celui des relations économiques en général, évolue sans cesse, avec des règles et des environnements économiques propres à chaque époque, mais avant de faire un tour d'horizon des principales étapes d'évolution du $\mathrm{SMI}^{2}$, il convient de bien le définir. Le SMI peut être pensé comme « un ensemble de conventions,

\footnotetext{
${ }^{1}$ Le SMI a connu de lourds phénomènes de hiérarchie et d'asymétrie entre monnaies. Il suffit de se référer au rôle de la livre sterling avant 1945, et celui de dollar américain après.

${ }^{2}$ Tout au long de ce papier on désignera le système monétaire international par son acronyme à savoir le SMI
} 
règles et instruments de politique économique qui déterminent les conditions et modalités de l'offre de liquidités internationales et de l'ajustement des déséquilibres extérieurs ; les régimes de change et de mouvements de capitaux ; les dispositifs de surveillance bilatéraux et régionaux et les instruments de prévention des crises ainsi que les modalités de leur résolution $»^{3}$.

\subsection{L'étalon-or et l'étalon change-or}

Le système de l'étalon-or a existé du milieu du XIX ${ }^{\mathrm{e}}$ siècle jusqu'aux années 20. En fait, il n'a jamais été un « choix », mais une fois l'économie mondiale a été lancée pendant la période de la domination économique de la Grande-Bretagne il a correspondu à une tentative mondialiste d'organisation des échanges, et du même coup des productions. Sous ce régime, les monnaies des pays concernés possédaient une équivalence fixe en or qui constituait l'instrument international de paiement; ainsi l'or circulait librement à l'intérieur des pays et les billets de banque pouvaient en être convertis sur demande. Les parités entre les monnaies étaient fixes, et les pays déterminaient le cours de leur propre monnaie, chaque pays maintenait des réserves d'or à partir desquelles, en principe, émettait des quantités de sa propre monnaie.

Le système de l'étalon-or s'est effondré en raison de la confusion engendrée de la première guerre mondiale, les pays participants ont abandonné la convertibilité-or des billets de banques et financé les dépenses de guerre par une augmentation de la masse monétaire, sans rapport avec leurs réserves en or, chose qui provoqua l'inflation et fini par entrainer la dévaluation de plusieurs monnaies notamment la livre sterling.

L'étalon change-or fut une transition vers le système de Bretton woods. La valeur des monnaies y était déterminée par rapport à une monnaie de référence, dont la valeur était définie par rapport à l'or. La livre sterling et le dollar américain étaient retenus comme monnaies de réserves, elles étaient convertibles en or à un taux fixe.

Cette transition entre deux systèmes a été une période de grands désordres monétaires, dès 1931, la Grande-Bretagne dévaluait sa monnaie et suspendait la convertibilité en refusant de verser l'or réclamé en échange des livres détenues à l'étranger. Le dollar américain était alors la seule devise convertible en or. La grande crise économique des années 30 fut suivie de la seconde guerre mondiale, face au désordre qui en a résulté, les États, au sortir de la guerre, ont cherché à poser les fondements d'une stabilité financière internationale.

\subsection{Le système de Bretton Woods}

L'effondrement du système de l'étalon change-or au cours des années 1930 a marqué les responsables de la reconstruction de l'après-guerre, À partir de 1942-43, britanniques et américains entamèrent des négociations afin de déterminer les principales caractéristiques de la nouvelle organisation monétaire international de l'après-guerre. Leurs positions étaient communes concernant les traits généraux du nouveau système, c'est-à-dire un système de changes fixes, qui assure le financement du commerce international et des mouvements des capitaux, tout en mettant en place une structure qui veille à l'application de ces nouvelles règles et qui garantisse l'approvisionnement en liquidité internationale. Cependant, dans le détail, la conception britannique différait sensiblement de l'américaine.

\footnotetext{
${ }^{3}$ Dorucci et Mckay, « The international monetary system after the financial crisis ». Occasional paper series n ${ }^{\circ} 123$, février 2011.
} 
La Conférence de Bretton Woods en 1944 a débouché sur un système monétaire international qui n'en était pas un car il ne reposait pas sur une vraie monnaie internationale. Le plan Keynes qui au contraire en prévoyait une n'ayant pas été adopté, ni même discuté. Comme les pays avaient besoin d'une monnaie pour régler leurs transactions, c'est le dollar qui a été utilisé et qui a pris peu à peu la place laissée vacante (Lelart, 2014). Il est important de signaler que les Accords de Bretton Woods avaient conféré au dollar un statut particulier. En effet, chaque pays doit définir la valeur externe de sa monnaie selon ses convenances soit par référence directe à l'or, soit par référence au dollar américain lui-même rattaché à l'or. Cette clause instaure un contexte où le dollar est considéré comme un substitut quasi parfait à l'or, Compte tenu de leurs réserves, les États-Unis était le seul pays capable de convertir sa monnaie en métal ${ }^{4}$. Le dollar devenait ainsi « as good as gold». Les accords de Bretton Woods ont aussi abouti à la création du Fonds Monétaire International (FMI) chargé de surveiller les politiques économiques nationales et de financer les déficits courants des pays (McKinnon, 1993 et Eichengreen, 2008) et à celle de la Banque Internationale pour la Reconstruction et le Développement (BIRD), plus communément appelée Banque Mondiale.

Le système de Bretton Woods s'est finalement effondré au début des années 1970 en conséquence de l'adoption par les États-Unis d'une politique très expansionniste, du caractère insoutenable de son déficit commercial et des pressions qui commençaient à s'exercer sur les taux de change fixes à cause du relâchement du contrôle des capitaux. Là encore, tous les pays ont en subi les répercussions. Avec la fin de la convertibilité du dollar en or et donc de l'ancrage sur l'or (1971) le système monétaire international a connu des mutations majeures. Notamment, le flottement généralisé des monnaies des pays avancés (1973), confirmés par les accords de la Jamaïque (1976), l'émergence d'une coopération monétaire européenne (1979), l'adoption temporaire d'une cible de change par quelques pays du G7 $(1985,1987)$ et enfin, la création et l'entrée en vigueur de l'euro $(1999,2002)$. La généralisation du modèle de la banque centrale indépendante, l'adoption par beaucoup d'entre elles de stratégies de ciblage de l'inflation et l'importance grandissante des économies émergentes, dont les préférences et les choix sont différents de ceux des économies avancées ${ }^{5}$. Au final, le SMI est devenu un système sans monnaie, les États-Unis peuvent toujours utiliser leur monnaie dans leurs transactions avec les autres pays. C'est bien pourquoi on parle d'un « non-système » (Camdessus et al, 2011) et on pourrait même, comme R. Triffin (1987), parler du SMI comme d'un « scandale monétaire international».

\section{Le portrait du SMI actuel}

Après la chute du système de Bretton Woods (figure ${ }^{\circ} 1$ ), le système monétaire international est retourné à un modèle plus décentralisé et davantage orienté sur les marchés. Les grands pays ont adopté des taux de change flottants, rendu leur monnaie convertible et libéralisé progressivement les flux de capitaux. Ces dernières années, plusieurs marchés émergents ont mis en place des politiques similaires après avoir éprouvé les difficultés associées à la gestion de régimes de changes fixes avec des comptes de capital de plus en plus ouverts. Le passage à des taux de change davantage déterminés par les marchés a eu pour effet d'accroître le contrôle de la politique monétaire intérieure et la maîtrise de l'inflation, d'accélérer le développement des secteurs financiers et, finalement, de stimuler la croissance économique. Le SMI qui s'est développé depuis les accords de la Jamaïque, en 1976, est hétérogène et

\footnotetext{
${ }^{4} \mathrm{Au}$ lendemain de la guerre, le stock d'or américain à Fort Knox s'élevait à 24 milliards de dollars

${ }^{5}$ Voir McKinnon (1993) pour une caractérisation des régimes de la seconde moitié du XXe siècle
} 
peut être qualifié de «semi-étalon dollar» (M. Aglietta, 2009) du fait de la coexistence des monnaies dans le cadre de divers régimes de change. Quelques monnaies relevant de grandes économies développées, fluctuent librement vis-à-vis du dollar. Cependant plusieurs des pays émergents lient leurs monnaies au dollar américain par des régimes de change qui vont des changes fixes au flottement géré. À l'heure actuelle, le SMI est un ensemble de politiques à visée nationale dans un contexte où les flux de capitaux sont essentiellement libres. Les États-Unis continuent de jouer un rôle central dans le système financier mondial. En plus de leurs marchés financiers profonds, le dollar demeure la principale devise internationale, à la fois pour les opérations commerciales et pour les réserves de change des banques centrales (I.Maes, 2010). Dooley, Folkerts-Landau et Garber (2003) décrivent même le système actuel comme une sorte de Bretton Woods « réactivé ».

Carney, (2009) et Lipsky, (2010) définissent le système monétaire international comme étant le résultat d'un ensemble de politiques et mécanismes officiels concernant la balance des paiements internationaux, plus exactement, les taux de change ; les paiements courants et les flux de capitaux ; et les réserves internationales. D'autant plus qu'il est constitué d'une panoplie d'institutions, de règlements, de normes et de conventions qui régissent son fonctionnement.

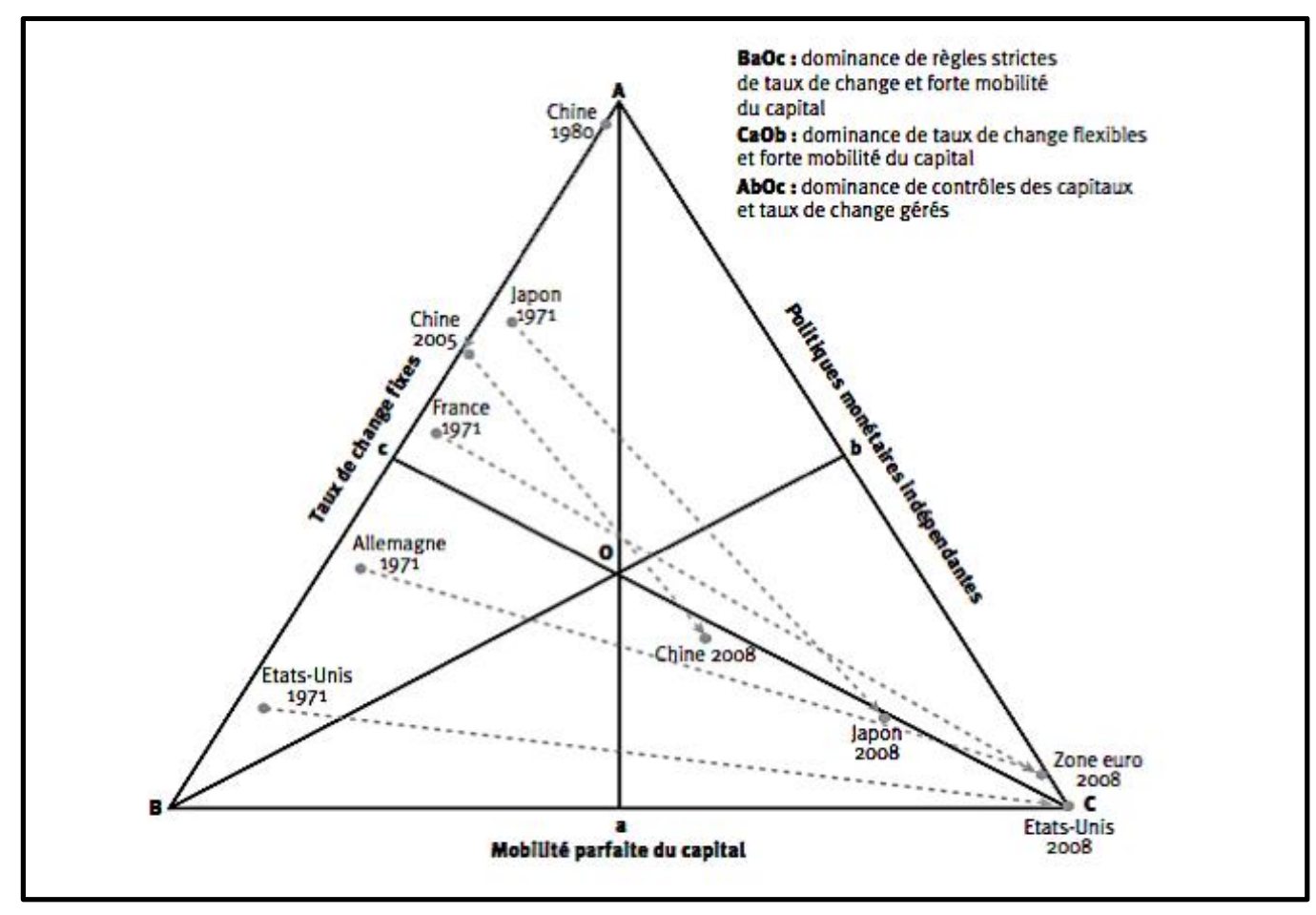

Figure 1 : l'évolution du SMI dans le cadre du dilemme de Mundell.

Source : Aglietta, M et al, 2010

\subsection{Taux et régimes de change}

La qualification du système monétaire international de système « hybride » est due au fait que les pays d'importance systémique n'ont en commun ni leur régime de change ni leur point d'ancrage nominal. Les monnaies relevant des deux tiers des 40 grandes économies développées fluctuent librement vis-à-vis du dollar (sur la foi du PIB fondé sur le marché), alors que le tiers restant, principalement des pays émergents, lient leurs monnaies au dollar américain par des régimes qui vont 
des changes fixes au flottement géré. Il reste important de souligner la persistance d'une «peur du flottement $»^{6}$ dans beaucoup d'économies émergentes ou en voie de développement.

\subsection{Paiements courants et flux de capitaux}

Généralement, la monnaie des pays avancés est convertible et leur compte de capital est ouvert. Cependant, les pratiques diffèrent du côté des économies émergentes, la tendance y était à la suppression des contrôles et à la libéralisation des paiements courants et des flux de capitaux. Pendant près de vingt ans et jusqu'au début des années 2000, ces soldes agrégés des balances courantes oscillaient entre $2 \%$ et un peu plus de $3 \%$ du PIB mondial. À partir de 2003 d'importants et persistants excédents et déficits des balances courantes ont été enregistrés, ces déséquilibres s'amplifient pour atteindre leur apogée en 2006 et 2007. En l'espace de quatre ans, ils ont doublé, passant de 3,3\% du PIB mondial en 2002 à 6,6 \% en 2006. Aux États-Unis, le déficit courant a atteint presque $6 \%$ du PIB en 2006, alors que l'excédent de la balance chinoise a culminé à 10,1\% du PIB en 2007(E. Santor et L. Schemri, 2011). Certes, La crise financière a contribué à l'atténuation de ces déséquilibres, mais c'est dû, en grande partie, au retournement cyclique de l'activité dans les pays avancés. Or le plus étonnant est la progression des flux de capitaux et du montant brut des actifs et passifs extérieurs. L'encours brut de ces derniers est en effet passé de $40 \%$ du PIB en 1970 à près de $300 \%$ en 2007 dans les pays du G20 (figure $\mathrm{n}^{\circ} 3$ ). Cette progression surprenante des flux bruts, qui est attribuable au mouvement de mondialisation financière, est l'un des principaux changements structurels qu'a connu le système monétaire international ces dernières années.

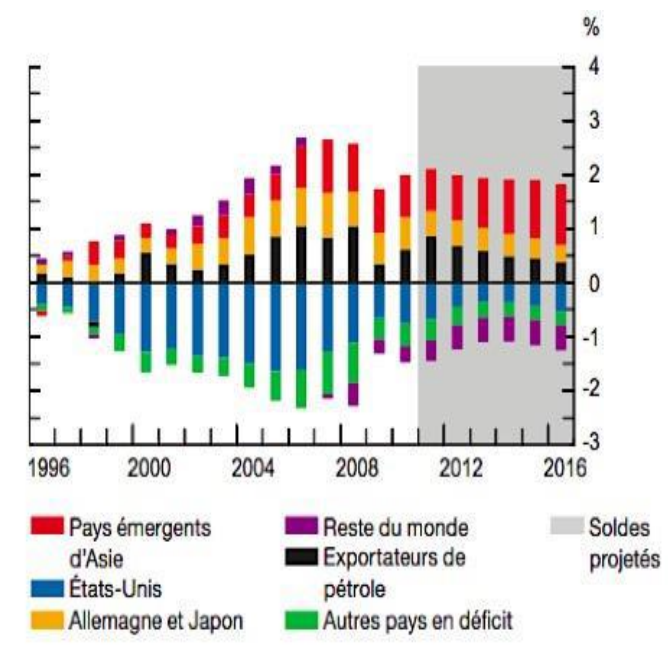

Figure $\mathbf{n}^{\circ} 2$ : Déséquilibres mondiaux ${ }^{7}$

Source : Conseil des gouverneurs de la Réserve fédérale américaine et estimations du FMI.

\footnotetext{
${ }^{6}$ Voir Calvo et Reinhart (2002)

${ }^{7}$ Solde de la balance courante en pourcentage du PIB mondial
}

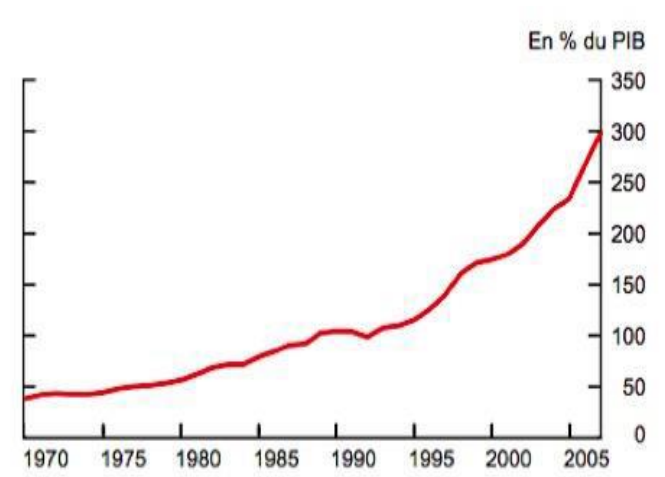

Figure $\mathbf{n}^{\circ} \mathbf{3}$ : Encours total brut des actifs et passifs extérieurs des pays du G20 Source : Santor, E et Schemri , L. 2011 


\subsection{Les réserves internationales}

Au lendemain du système de Bretton Woods, plusieurs pays développés ayant une monnaie flottante ont diminué leur niveau des réserves par rapport à leur PIB. À l'opposé, ces dernières années, on a assisté à une importante accumulation des avoirs de réserve dans les marchés émergents ${ }^{8}$ conjuguée avec l'accentuation des déséquilibres des comptes courants. Les réserves internationales, qui se chiffraient à moins de 1 billion de dollars américains en 2000, ont totalisé près de 7 billions en 2011 et sont passées à 10.9 billions de dollars en 2015 (figures $n^{\circ} 4$ et 5) dont la majeure partie est investie en titres de dette de l'État américain. On note bien la part prépondérante du dollar dans les réserves mondiales qui atteint près de $64 \%$ et celle de l'euro de 19,9\%. La livre sterling et le yen représentaient chacun 4,9\%. Officiellement, la part du renminbi (RMB) n'apparaîtra que dans les statistiques officielles de 2016, le FMI a décidé d'inclure le RMB dans le panier de Droits de tirage spéciaux (DTS). Le niveau des réserves mondiales dépasse largement le niveau dicté pour des motifs de précaution. La taille et la persistance des déséquilibres des balances courantes et l'accumulation résultante de réserves colossales sont la conséquence directe de l'absence d'ajustements opportuns et symétriques des taux de change réels au sein du système monétaire international (E. Santor et L. Schemri, 2011).
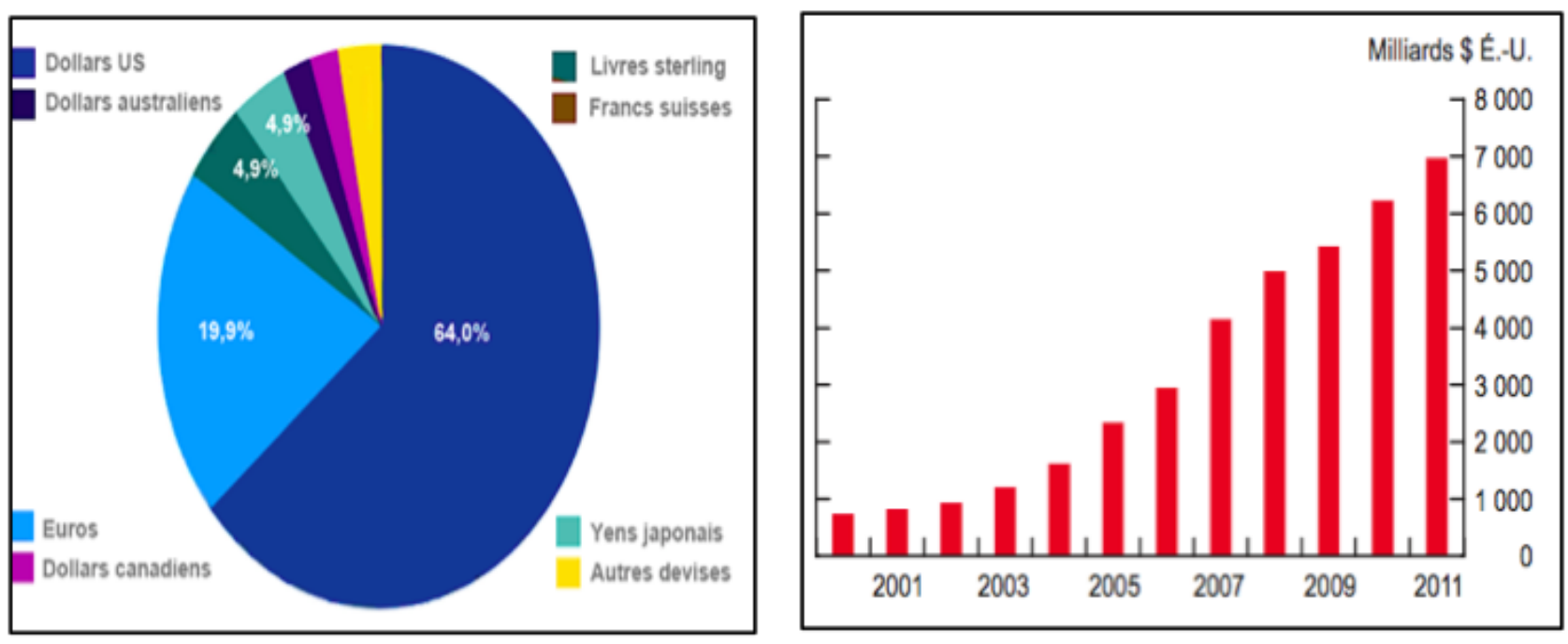

Figure $n^{\circ} \mathbf{4}$ : les réserves mondiales allouées par devises pour le T4 2015

Source : FMI, Statistiques financières internationales
Figure ${ }^{\circ} \mathbf{5}$ : Réserves internationales des économies émergentes et en développement

Source : FMI, Statistiques financières internationales

\footnotetext{
${ }^{8}$ Certaines grandes économies ont accumulé des quantités énormes de réserves de change dans l'optique de se protéger contre les crises futures
} 


\subsection{Les instances monétaires}

La surveillance du système monétaire international relève d'un ensemble d'institutions dont L'objectif est de préserver la stabilité financière et monétaire dans le monde en mettant en œuvre une panoplie de règles, normes et ententes ressortissant aux politiques macroéconomiques et à la réglementation financière. Les principales institutions chargées de superviser le système monétaire (figure ${ }^{\circ} 6$ ), à savoir, le FMI, la Banque des Règlements Internationaux (BRI), le Conseil de stabilité financière (CSF) et le G20 avaient cerné déjà bon nombre des risques qui se sont matérialisés pendant la crise de 2007-2009. Cependant, leur efficacité reste contestable.

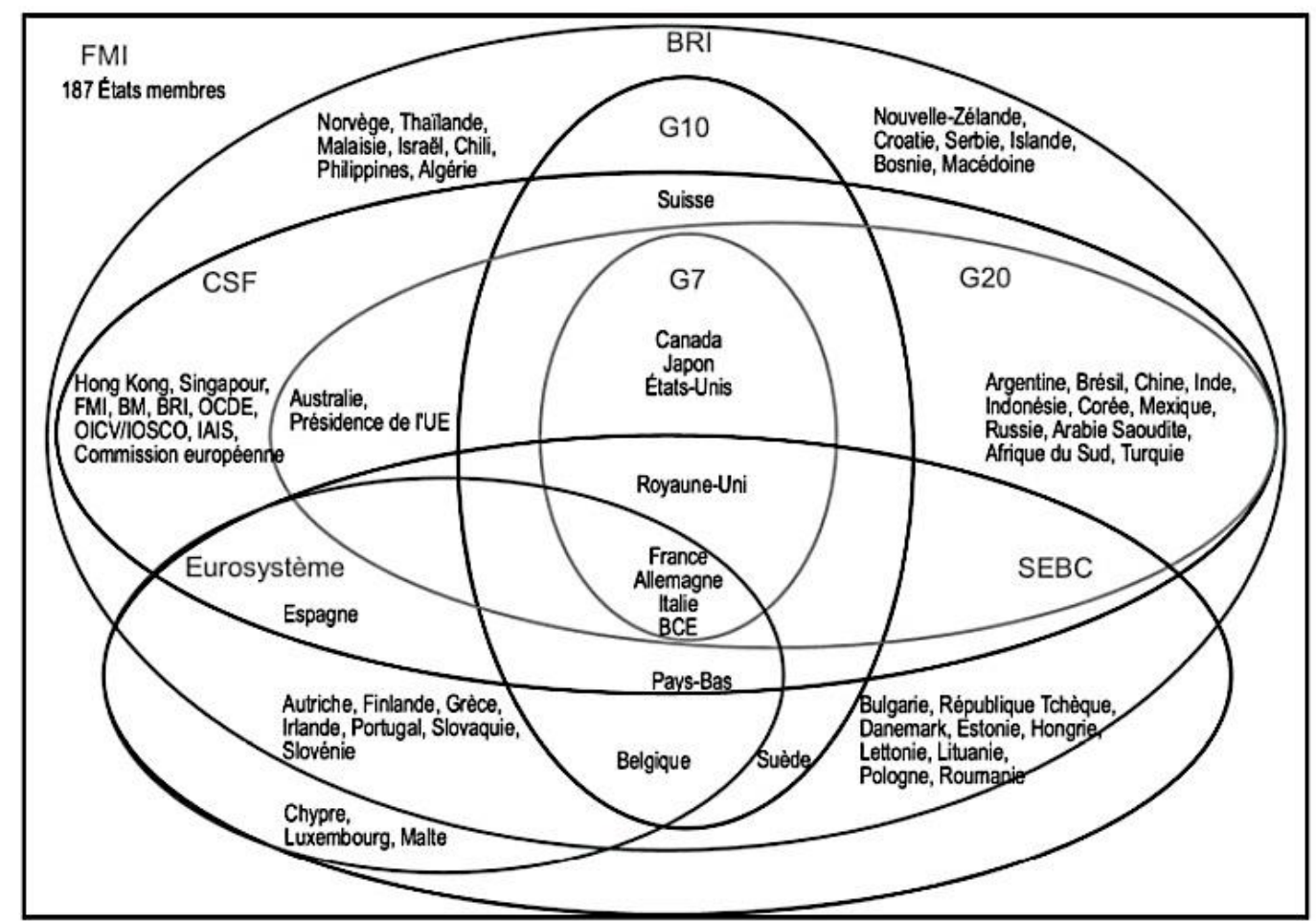

Figure $\mathbf{n}^{\circ} \mathbf{6}$ : les différentes institutions monétaires internationales

\section{Le déclin du SMI actuel}

Le système monétaire international actuel a favorisé l'essor faramineux de l'activité économique, des échanges commerciaux et de l'intégration financière à l'échelle mondiale. En effet, depuis 1970, le PIB mondial a crû annuellement de plus de $3 \%$ en moyenne ; le commerce entre pays, de près de $6 \%$; et le montant brut des actifs et passifs extérieurs, de plus de $9 \%$ (E. Santor et L. Schemri, 2011). Ces résultats sont allés de pair avec l'intégration de la Chine et de l'Inde à l'économie mondiale. Le commerce international et les investissements directs étrangers ont permis à la Chine non seulement de bénéficier d'un accès aux marchés, de transferts de technologie et d'une spécialisation accrue, mais aussi de tirer parti de l'avantage comparatif qu'elle possède dans la fabrication de produits à forte 
intensité de main-d'œuvre. D'une manière générale, le SMI est bien parvenu à appuyer la croissance des échanges commerciaux et des flux de capitaux et ce malgré les crises bancaires, de dette souveraine et de change qu'il a dû affronter depuis l'abandon des accords de Bretton Woods.

Aujourd'hui, les débats sur les inconvénients de tel ou tel système monétaire se concentrent sur quelques points centraux que nous aborderons ci-après. Théoriquement, le fait que les pays n'adoptent pas tous le même point d'ancrage nominal pour la conduite de leur politique monétaire (certains optant pour la stabilité des prix, d'autres pour celle des taux de change) ne constitue pas forcement une atteinte à la stabilité du système monétaire international, dans la mesure où les taux de change réels puissent s'ajuster en réaction aux chocs. Sans un engagement des pays en ce sens, le système actuel se heurte à certaines asymétries fondamentales.

Pour Jacques Mistral, (2014), depuis l'entrée de la Chine comme puissance économique mondiale, la coexistence entre changes flottants et changes fixes fait du SMI un " non-système » monétaire international car elle s'accompagne de déséquilibres des Balances des paiements de plus en plus problématique pour la stabilité du système. Selon Aglietta (2010), il y a deux principaux déséquilibres. D’une part le « déséquilibre épargne / investissement » vu que c'est l'excédent d'épargne dans les pays émergents qui finance les déficits courants dans les $\mathrm{PDEM}^{9}$, principalement les États-Unis, et d'autre part «l'explosion de la liquidité [...] des années $2000 »$, puisque les marchés financiers ne régulent pas les excès d'épargne. Chose qui mène à l'instabilité du SMI. Bénassy-Quéré et Pisani-Ferry (2011), reviennent sur ce qu'ils ont appelé «les défauts du SMI». Dans leur analyse, ils développent cinq principales anomalies/ défauts du système actuel. Les désajustements de change, la recherche sur les effets des fluctuations de change a montré que la volatilité de court terme des taux de change était finalement moins dommageable que les désajustements marqués et persistants, lesquels conduisent à des distorsions dans les choix économiques (investissement, épargne, emploi, développements sectoriels $)^{10}$, la volatilité des mouvements de capitaux et les moyens d'y faire face. Au final, la libéralisation financière n'a pas eu les résultats escomptés. Car, Au lieu de favoriser la stabilité macroéconomique en permettant l'absorption de chocs temporaires sur le revenu, elle s'est accompagnée d'une grande volatilité des flux et a donc souvent été considérée comme facteur d'instabilité (Kose, Prasad, Rogoff et Wei, 2006). La volonté des pays d'intervenir sur le marché des biens et l'incertitude sur l'accès à la liquidité ou sur les conditions associées à sa fourniture ont conduit à une importante accumulation de réserves dans les pays émergents pour servir ainsi à une sorte d'auto-assurance.

\footnotetext{
${ }^{9}$ PDEM : pays développés à économie de marché

${ }^{10}$ Cet argument a longtemps été utilisé contre les changes flottants, Aujourd'hui, c'est devenu un argument contre la fixité des taux de change qui retarde les ajustements de taux de change réels
} 
Le quatrième défaut, toujours selon Bénassy-Quéré et Pisani-Ferry (2011) est l'absence d'une discipline et d'une coordination des politiques macroéconomiques. Il y a, enfin le problème de l'orientation de la politique monétaire au niveau mondial. Le rôle essentiel du SMI est de s'assurer de la cohésion des politiques monétaires nationales avec la stabilité des prix au niveau mondial. Ce rôle était passé au second plan dans le contexte de la «grande modération » des années quatre-vingt-dix et 2000, mais revient au premier plan dans un contexte de contraintes de ressources.

Dans ce cadre le système monétaire international a été considéré partiellement responsable de la crise, parce qu'il aurait favorisé un afflux de capitaux et la formation d'une bulle de crédit aux États-Unis. Au total, le régime monétaire international actuel est le produit d'une série d'évolutions résultant de choix opérés individuellement par un grand nombre de pays. Certains de ses défauts sont connus de longue date et son rôle dans la genèse de la crise est controversé, mais il est important de préciser qu'il a fonctionné de façon satisfaisante une fois celle-ci déclenchée. Au final, le régime actuel est mal adapté au nouveau contexte économique de l'après-crise. L'ensemble des déséquilibres et faiblesses du SMI illustrent bien la pertinence et la nécessité de sa réforme.

\section{Vers un nouveau SMI}

En considérant la persistance des déséquilibres, des tensions sur les marchés des changes et des vulnérabilités observées, le SMI doit impérativement être reconsidéré afin de permettre des ajustements plus symétriques et plus rapides des taux de change, qui auront tendance à faciliter le rééquilibrage des flux du commerce extérieur et des flux de capitaux, et à anticiper la réapparition de déséquilibres extérieurs profonds.

La transformation du SMI relève de trois scénarios envisageables, Le premier, selon C.Kindleberger et Arvind Subramanian, consiste à imaginer qu'une nation joue un rôle hégémonique. Si le SMI actuel ne fonctionne plus bien c'est parce que les États-Unis ont perdu leur statut de puissance hégémonique mais qu'aucun autre pays ne les remplace encore. Il faudrait imaginer que, par exemple, la Chine s'impose à la fois économiquement et politiquement sur la scène mondiale. Sauf que la théorie de la stabilité hégémonique présente ainsi deux limites importantes (Bénassy-Quéré et Pisani-Ferry, 2011). Dans un premier temps, elle considère que la «puissance hégémonique » jouit d'une prédominance économique incontestée, et a donc tout intérêt à assurer et préserver la stabilité internationale. Et dans un second temps, cette théorie néglige la possibilité, pour la puissance en question, d'exploiter son pouvoir de monopole ; autrement dit, d'utiliser son " privilège exorbitant » à des fins purement nationales, au détriment de la stabilité internationale.

Le second scénario est l'approche la plus privilégiée par les organisations internationales ((FMI), les États-Unis et l'Europe), elle est fondée sur une amélioration de la surveillance multilatérale et sur un accès plus facile à la liquidité en temps de crises, cela implique de faire évoluer le FMI, dont le rôle est 
en débat depuis des années. Désormais, un consensus assez large se dégage pour réformer la gouvernance du FMI. Trevor Manuel (2009) propose que les quotas doivent être modifiés afin de donner plus de poids aux pays émergents et refléter les réalités du XXIe siècle. Le Comité économique et financier international doit être transformé en un véritable Conseil réunissant ministres et gouverneurs pour devenir, conformément aux statuts, un organe de responsabilité politique à la tête du Fonds en charge en particulier des décisions stratégiques ; «l'executive board» actuel doit se concentrer sur la surveillance macroéconomique, sur les décisions financières du Fonds et sur la supervision de l'action du management.

Quant au troisième scénario, il est fondé sur le paradoxe de Triffin adapté à la période contemporaine. La capacité des États-Unis à fournir la liquidité mondiale va tôt ou tard se heurter aux besoins de l'économie mondiale, on va donc assister à une pénurie de dollars. En effet, pour que du dollar puisse circuler dans le monde et assurer son rôle de monnaie internationale, les États-Unis doivent émettre des titres libellés en dollar. On parle de dilemme de Triffin parce que, finalement, soit les USA sont capables d'émettre beaucoup de dollars, mais cela se fait au prix de l'accroissement de l'endettement extérieur au risque de devenir insoutenable ; soit les USA limitent l'émission de dette et donc de dollar, mais à ce moment là la pénurie de dollar pousse ceux qui en ont besoin à trouver des solutions alternatives. Cette «pénurie » de dollar peut par ailleurs survenir à un moment de régionalisation des échanges. Or, c'est bien ce qui est entrain de se générer ; l'intégration mondiale a tendance à reculer au profit d'une intégration régionale plus forte (Amérique du nord, Europe, Asie). Le résultat de l'émergence de ce monde multipolaire est le développement de monnaies concurrentes : en l'occurrence, l'euro et le yuan. Comme l'économie se régionalise, chaque région va posséder sa devise clé. Sauf que, il existe bel et bien une solution « optimale », il s'agit de celle préconisée par certains économistes (Aglietta, Mistral, rapport du Cepii et du CAE) elle consiste à adopter une monnaie internationale qui ne soit pas une devise nationale. Cette idée défendue en 1944 par Keynes (le bancor), dans un contexte de contrôle des mouvements de capitaux, pourrait être relancée aujourd'hui dans un contexte de globalisation financière en utilisant les DTS (droits de tirages spéciaux) émis par le FMI. Les DTS sont qualifiés d' « instrument de réserve ultime », Il y a trois raisons pour le renforcement de leur rôle. La première consiste à atténuer les insuffisances du semi-étalon dollar en limitant l'acquisition du dollar par les banques centrales. La deuxième est d'allouer suffisamment de réserves dans le but d'éviter l'accumulation des réserves de précaution en dollar par les pays dont les monnaies sont faiblement convertibles. La troisième est la création d'un compte de substitution au sein du FMI, afin de permettre aux pays disposant d'une quantité excessive de réserves en dollar de diversifier la répartition de leurs réserves en dehors du marché des changes, et d'éviter de fortes variations perturbatrices des taux de change (Aglietta, 2010). 
Le processus de mondialisation a été bénéfique pour l'économie mondiale avec plus de croissance, plus d'échanges, des ajustements qui s'avéraient difficiles, certes mais avec des opportunités inouïes. Sauf qu'en contrepartie, elle a dû faire face à l'aggravation des déséquilibres globaux, la création incontrôlée de liquidités, la concurrence et paradoxalement la complémentarité entre différentes sortes de capitalismes. Depuis l'irruption de la Chine, le contraste est saisissant en matière monétaire où coexistent un régime de changes flexibles, en particulier entre le dollar et l'euro, et la recherche d'un change stable en particulier pour le yuan, ce qui a produit un basculement vers le «non-système ». Les scénarios de réforme cités plus haut s'inscrivent dans la même logique endogène que les SMI qui se sont succédés jusque-là. Il faut tirer des leçons de l'histoire pour envisager et mettre en œuvre des solutions qui répondent, entre autres, à trois critères, en effet la puissance économique se déplace du G7 vers les économies émergentes, Il faut, d'abord, que ce système reflète dans ses institutions et ses dispositifs opérationnels la logique des forces économiques et financières aujourd'hui à l'œuvre, une logique signée par un pluralisme de systèmes économiques dont il faut agencer les relations. Ensuite, il doit traduire les réalités géopolitiques en prenant en compte de manière équilibrée le poids et les intérêts des principaux acteurs. Enfin, il faut qu'il soit accepté par tous (ou presque) et qui suit l'état des idées et doctrines en matière financière, une sorte de jonction entre forces de marché et responsabilités des gouvernements. Or, le SMI actuel repose sur le principe que les déséquilibres mondiaux se corrigeront spontanément dans le cadre des régimes de change flexibles, sachant que même s'il continue de fonctionner avec un petit nombre de monnaies de réserve, la part des échanges mondiaux opérés par des pays dont la monnaie n'est pas une monnaie de réserve continue d'augmenter, ce qui déstabilise davantage le système. D'où la proposition d'utiliser les DTS de la même manière que le dollar aujourd'hui, il est vrai qu'ils ont l'avantage d'être supra-souverains mais leur caractère virtuel les affaiblit en tant que garantie, de même il est difficile de penser à remplacer le dollar par les DTS compte tenu de leurs limites en termes de disponibilité et d'utilisation à des fins privées, il s'agit d'une créance sur un panier de devises et non une devise au sens propre. Historiquement, les DTS ne représentaient qu'une maigre fraction des réserves mondiales 8,4\% de réserves autres que l'or en 1972 et moins de $3 \%$ au cours des dernières années d'autant plus qu'il existe peu de possibilités de placement dans cet instrument. Même la piste d'un éventuel retour au système étalon-or a été évoquée, sachant qu'en période de crise, statut de valeur refuge oblige, le cours du métal jaune ne cesse d'augmenter, surtout, après que Robert Zoellick a proposé d'employer de l'or comme point de référence international des anticipations des marchés au point de vue de l'inflation, de la déflation et des taux de change futurs, sauf qu'un tel système nécessite, entre autres, une production d'or qui puisse croître aussi rapidement que les échanges mondiaux, ce qui n'est pas le cas aujourd'hui. 


\section{Conclusion}

Il faut souligner que la crise de 2008/2009 offre une opportunité exceptionnelle de réforme et de refonte du SMI. L'ampleur des dégâts invite à redessiner les contours de la finance internationale pour qu'elle assure ses fonctions sans porter préjudice à l'économie réelle. La stabilisation du SMI est non seulement souhaitable, mais également indispensable à un rétablissement de la croissance mondiale et au financement du développement des pays du Sud.

Tout au long de ce papier nous avons analysé le SMI et son évolution dans le temps avec ses différentes réformes, pour répondre à chaque fois aux besoins de l'économie mondiale de l'époque, nous avons examiné ses forces et ses faiblesses pour s'atteler ensuite aux différentes pistes de réforme pour un «nouveau système monétaire international ». Le monde se dirige de plus en plus vers un polycentrisme monétaire à travers la constitution de vastes régions de coopération monétaire. Nous avons présenté certaines contributions des auteurs les plus actifs dans les débats autour de la réforme du SMI, les prévisions et les projets de réforme sont multiples. Qu'il s'agisse d'une réforme du FMI, d'un nouveau rôle du DTS, d'une relance du plan Keynes ou simplement de la maîtrise des déficits courants sauf qu'aucun de ces projets ne semble avoir pour l'instant quelque chance d'aboutir.

\section{BIBLIOGRAPHIE}

[1] AGLIETTA, M. (2008), « La gouvernance du système monétaire international », Regards croisés sur l'économie ( $\left.\mathrm{n}^{\circ} 3\right)$, p. $276-285$.

[2] AGLIETTA, M. (2010), « Le dollar, le yuan et le système monétaire international », L'Économie politique $\left(n^{\circ} 45\right)$, p. 6-25.

[3] BARREDO, J-M et ZURIARRAIN. (2015), «Le Système Monétaire International comme facteur d'instabilité : une ébauche de réforme ».

[4] BÉNASSY-QUÉRÉ, A et PISANI-FERRY, J. (2011), «Quel système monétaire international pour une économie mondiale en mutation rapide? »

[5] BÉNASSY-QUÉRÉ, A. (2012), « Le système monétaire international a besoin de l'euro », les Échos.

[6] BIBEAU, J-P. introduction à l'économie internationale, 4ème édition.

[7] CALVO, G et RENHART, C. (2002), «Fear of Floating », Quarterly Journal of Economics, vol. CXVII, $n^{\circ}$ 2, mai, pp. 379-408

[8] CAMDESSUS, M. LAMFALUSSY, A. PADOA-SCHIOPPA,T. et alii, (2011), « La réforme du système monétaire international : une approche coopérative pour le XXIème siècle », rapport du groupe « Initiative du Palais-Royal », Paris.

[9] DOOLEY, M. FOLKERTS-LANDAU, D et GARBER, P. (2003), «An essay on the revived Bretton Woods System », National Bureau of Economic Research, Working Paper 9971.

[10] Eichengreen, B. (2008), «Globalizing Capital. A History of the International Monetary System », Princeton University Press (2e édition)

[11] FARHI, E. GOURINCHAS, P-O. et REY, H. (2011), «Quelle réforme pour le système monétaire international?»

[12] JACKE, P. (1994), « le système monétaire international en devenir » 
[13] KOSE, M-A. PRASAD, E. ROGOFF, K ET WEI, S-J. (2006), « Financial Globalisation: A Reappraisal », IMF Working Paper, vol. 56, $\mathrm{n}^{\circ} 1$.

[14] Le 85ème rapport annuel de la Banque des règlements internationaux

[15] LELART, M. (2014), « Le système monétaire international face aux critères du développement durable »

[16] Lipsky, J. (2010), « Reconsidering the International Monetary System », Macroeconomic Challenges: The Decade Ahead, actes du symposium annuel tenu par la Banque fédérale de réserve de Kansas City à Jackson Hole (Wyoming).

[17] M. CARNEY, M. (2009), « L’évolution du système monétaire international », discours prononcé devant la Foreign Policy Association.

[18] MAES, I. (2010), « La genèse du système monétaire international actuel », Reflets et perspectives de la vie économique (Tome XLIX), p. 17-27.

[19] MANUEL, T. (2009), le rapport du comité sur la réforme de la gouvernance du FMI

[20] MCKINNON, R. (1993), «The Rules of the Game: International Money in Historical Perspective, » Journal of Economic Literature, vol. 31, $\mathrm{n}^{\circ}$ 1, mars, pp. 1-44.

[21] MISTRAL, J. (2011), «La réforme du système monétaire international : une approche d'économie politique »

[22] MISTRAL, J. (2014), « Guerres et paix entre les monnaies ».

[23] PONSOT, J-F. (2013), «Les quatre I du système monétaire international et l'internationalisation de l'euro »

[24] SANTOR, E et SCHEMBRI, L. (2011), «Le système monétaire international : évaluation et pistes de réforme »

[25] Triffin, R. (1987). Le SMI (Systéme... Scandale ? Monétaire International) et le SME (Système Monétaire Européen). In R. Barre, A. Dunkel, G. Gaudard, A. Lamfalussy, J. L’Huillier, H. Mercillon, ... R. Triffin, Les déséquilibres monétaires et financiers extérieurs (pp. 31-56).

[26] UNGER, E. (2016), « La réforme du système monétaire mondial » 